Journal of Mathematical Physics, Analysis, Geometry

2017, vol. 13, No. 3, pp. 254-267

doi:10.15407/mag13.03.254

\title{
Approximate Solving of the Third Boundary Value Problems for Helmholtz Equations in the Plane with Parallel Cuts
}

\author{
V.D. Dushkin \\ National Academy of the National Guard of Ukraine \\ 3 Zakhystnykiv Ukrainy Sq., Kharkiv, 61001, Ukraine \\ E-mail:dushkinvd@gmail.com
}

Received October 10, 2015, revised February 6, 2017

\begin{abstract}
In the paper, the method of approximate solution of boundary integral equations of the original problem is proposed. The systems of boundary integral equations of the problem are obtained by the method of parametric representation of integral transforms. The convergence of approximate solutions to the exact solution of the original problem is guaranteed by the propositions proved in the paper. Also, the rate of convergence of approximate solutions to exact solutions is found.

Key words: approximate solution of boundary integral equations, singular integral equation, existence of approximate solution, rate of convergence of approximate solution.
\end{abstract}

Mathematical Subject Classification 2010: 45P05, 45L05.

\section{Introduction}

The modeling of electromagnetic waves diffraction on non-perfectly conducting gratings leads to the consideration of external mixed boundary value problems for the Helmholtz equation [1].

Modern diffraction structures consist of large number of elements located in different planes. It leads to the consideration of boundary value problems in domains of complex shape. An effective way of solving these boundary value problems was proposed by $\mathrm{Yu}$.V. Gandel. It consists of two basic steps. First, the initial boundary value problems are reduced to a system of boundary integral equations by the method of parametric representations of integral transforms [2-4]. Usually the systems of boundary integral equations consist of integral

(C) V.D. Dushkin, 2017 
equations of different types: the first and second kind, singular and hypersingular $[5,6]$. Second, finite-dimensional approximations of systems of boundary integral equations are constructed by using the method of discrete singularities (the method of discrete vortices [7]).

Methods of approximate solution of the first and second boundary value problems were well studied in [8-10]. Unfortunately, the theory of finding approximate solutions of boundary integral equation systems of the third boundary value problems has not been sufficiently studied yet because of the complexity of these systems. In particular, several types of equations depend on the same unknown functions. This makes it impossible to solve the equations independently from each other. Also, the presence of components with variable upper limit in the integral equations makes it difficult to apply the classical scheme of the method of discrete singularities.

In this article, a version of the approximate solution of boundary integral equations of the third boundary value problem, which was considered in [11], was proposed. The version is based on the ideas of $[12,13]$, where the approximate solution of the diffraction problem of electromagnetic waves on a system of superconductive bands [14] was considered.

\section{System of Boundary Integral Equations}

The system of boundary integral equations of the initial problem consists of integral equations of two different types.

Equations of the first type are singular integral equations of the first kind:

$$
\begin{aligned}
\frac{1}{\pi} \int_{-1}^{1} \frac{1}{\tau-\xi} \frac{\nu_{q}(\tau) d \tau}{\sqrt{1-\tau^{2}}} & +\frac{1}{\pi} \sum_{p=1}^{2 R} \int_{-1}^{1} Q_{q, p}(\xi, \tau) \frac{\nu_{p}(\tau) d \tau}{\sqrt{1-\tau^{2}}} \\
& -\frac{c_{q}}{\pi} \int_{-1}^{\xi} \frac{\nu_{q}(\tau) d \tau}{\sqrt{1-\tau^{2}}}=f_{q}(\xi), \quad|\xi|<1, \quad q=1, \ldots, R
\end{aligned}
$$

with the additional conditions

$$
\frac{1}{\pi} \int_{-1}^{1} \frac{v_{q}(\tau) d \tau}{\sqrt{1-\tau^{2}}}=0, \quad q=1, \ldots, R .
$$

Equations of the second type are Fredholm equations of the second kind:

$$
\nu_{q}(\xi)+\frac{1}{\pi} \sum_{p=1}^{2 R} \int_{-1}^{1} Q_{q, p}(\xi, \tau) \frac{\nu_{p}(\tau) d \tau}{\sqrt{1-\tau^{2}}}
$$




$$
-\frac{c_{q}}{\pi} \sqrt{1-\xi^{2}} \cdot \int_{-1}^{\xi} \frac{\nu_{q-R}(\tau) d \tau}{\sqrt{1-\tau^{2}}}=f_{q}(\xi), \quad|\xi|<1, q=R+1, \ldots, 2 R .
$$

In equations (1), (3) it is assumed that

$$
\begin{array}{r}
f_{q}(\xi) \in C^{0, \frac{1}{2}}[-1,1], \quad Q_{q, p}(\xi, \tau) \in C^{0, \frac{1}{2}}([-1,1] \times[-1,1]), \\
p=1, \ldots, 2 R, \quad q=1, \ldots, 2 R .
\end{array}
$$

In these equations $R$ is the number of plane-parallel slits.

Let $L_{\rho, \alpha}^{2},(\alpha= \pm 1)$, be the Hilbert spaces of measurable functions with respect to the inner product

$$
(u, \nu)_{\alpha}=\int_{-1}^{1} u(\tau) \bar{v}(\tau) \rho^{\alpha}(\tau) d \tau, \quad \rho(\tau)=\sqrt{1-\tau^{2}},
$$

and the norm $\|\nu\|_{\alpha}=\sqrt{(v, \nu)_{\alpha}}$. Also we take under consideration the space

$$
L_{\rho,-1}^{2,0}=\left\{u \in L_{\rho,-1}^{2} \mid(u, 1)_{-1}=0\right\} .
$$

Define the operators:

$$
\begin{aligned}
& \Theta_{q, p}: L_{\rho,-1}^{2} \rightarrow L_{\rho, 1}^{2}, \quad\left(\Theta_{q, p} u\right)(\xi)=\frac{1}{\pi} \int_{-1}^{1} Q_{q, p}(\xi, \tau) \frac{u(\tau) d \tau}{\sqrt{1-\tau^{2}}}, \\
& |\xi|<1 ; \quad q=1, \ldots, R ; \quad p=1, \ldots, 2 R \\
& \Theta_{q, p}: L_{\rho,-1}^{2} \rightarrow L_{\rho,-1}^{2}, \quad\left(\Theta_{q, p} u\right)(\xi)=\frac{1}{\pi} \int_{-1}^{1} Q_{q, p}(\xi, \tau) \frac{u(\tau) d \tau}{\sqrt{1-\tau^{2}}} \\
& |\xi|<1 ; \quad q=R+1, \ldots, 2 R ; \quad p=1, \ldots, 2 R ; \\
& \Phi: L_{\rho,-1}^{2,0} \rightarrow L_{\rho, 1}^{2}, \quad(\Phi u)(\xi)=\frac{1}{\pi} \int_{-1}^{\xi} \frac{u(\tau) d \tau}{\sqrt{1-\tau^{2}}}, \quad|\xi|<1 \\
& \Psi: L_{\rho,-1}^{2,0} \rightarrow L_{\rho,-1}^{2}, \quad(\Psi u)(\xi)=\frac{\sqrt{1-\xi^{2}}}{\pi} \int_{-1}^{\xi} \frac{u(\tau) d \tau}{\sqrt{1-\tau^{2}}}, \quad|\xi|<1 \\
& \Gamma: L_{\rho,-1}^{2,0} \rightarrow L_{\rho, 1}^{2}, \quad(\Gamma u)(\xi)=\frac{1}{\pi} \int_{-1}^{-1} \frac{1}{\tau-\xi} \frac{u(\tau) d \tau}{\sqrt{1-\tau^{2}}}, \quad|\xi|<1 .
\end{aligned}
$$


From [15], it is known that

$$
\begin{array}{ll}
\Gamma: T_{0}(\tau) \rightarrow 0, \quad T_{n}(\tau) \rightarrow U_{n-1}(\xi), & n \in N ; \\
\Phi: T_{n}(\tau) \rightarrow-\frac{U_{n-1}(\xi)}{n} \sqrt{1-\xi^{2}}, & n \in N,
\end{array}
$$

where $\mathrm{T}_{n}(\tau)$ are the Chebyshev polynomials of the first kind, and $U_{n}(\xi)$ are the Chebyshev polynomials of the second kind.

Combining (7) and (10), we get

$$
\Psi: T_{n}(\tau) \rightarrow-\frac{U_{n-1}(\xi)}{n}\left(1-\xi^{2}\right), \quad n \in N
$$

and

$$
\|\Gamma\|_{L_{\rho,-1}^{2,0} \rightarrow L_{\rho, 1}^{2}}=1, \quad\|\Psi\|_{L_{\rho,-1}^{2,0} \rightarrow L_{\rho,-1}^{2}} \leq 1, \quad\|\Phi\|_{L_{\rho,-1}^{2,0} \rightarrow L_{\rho, 1}^{2}} \leq 1 .
$$

We introduce the Hilbert spaces $H_{1}^{2}$ and $H_{2}^{2}$ :

$$
\begin{aligned}
\vec{V} & =\left(v_{1}, v_{2}, \ldots, v_{2 R}\right) \in H_{1}^{2} \\
& \Leftrightarrow\left(v_{q} \in L_{\rho,-1}^{2,0}, \quad q=1, \ldots, R ; \quad v_{q} \in L_{\rho,-1}^{2}, \quad q=R+1, \ldots, 2 R\right) ; \\
\vec{W} & =\left(w_{1}, w_{2}, \ldots, w_{2 R}\right) \in H_{2}^{2} \\
& \Leftrightarrow\left(w_{q} \in L_{\rho, 1}^{2}, \quad q=1, \ldots, R ; \quad w_{q} \in L_{\rho,-1}^{2}, \quad q=R+1, \ldots, 2 R\right),
\end{aligned}
$$

with the inner products

$$
\begin{aligned}
& (\vec{V}, \vec{U})_{H_{1}^{2}}=\sum_{i=1}^{2 R}\left(v_{i}, u_{i}\right)_{L_{\rho,-1}^{2}} \\
& (\vec{W}, \vec{S})_{H_{2}^{2}}=\sum_{i=1}^{R}\left(v_{i}, s_{i}\right)_{L_{\rho, 1}^{2}}+\sum_{i=R+1}^{2 R}\left(v_{i}, s_{i}\right)_{L_{\rho,-1}^{2}}
\end{aligned}
$$

and the norms $\|\vec{V}\|_{H_{1}^{2}}=\sqrt{(\vec{V}, \vec{V})_{H_{1}^{2}}},\|\vec{W}\|_{H_{2}^{2}}=\sqrt{(\vec{W}, \vec{W})_{H_{2}^{2}}}$.

Consider the operators:

$$
\begin{aligned}
\mathbf{G}: H_{1}^{2} \rightarrow H_{2}^{2}, & (\vec{W}=\mathbf{G} \vec{V}) \\
& \Leftrightarrow\left\{\begin{array}{ll}
w_{q}=\Gamma v_{q}, & q=1, \ldots, R \\
w_{q}=v_{q}, & q=R+1, \ldots, 2 R
\end{array}\right\} ; \\
\mathbf{Z}: H_{1}^{2} \rightarrow H_{2}^{2}, \quad(\vec{W}=\mathbf{Z} \vec{V}) & \Leftrightarrow\left\{\begin{array}{ll}
w_{q}=c_{q} \Phi v_{q}, & q=1, \ldots, R \\
w_{q}=c_{q} \Psi v_{q-R}, & q=R+1, \ldots, 2 R
\end{array}\right\} ;
\end{aligned}
$$




$$
\begin{aligned}
\mathbf{K}: H_{1}^{2} \rightarrow H_{2}^{2}, & (\vec{W}=\mathbf{K} \vec{V}) \\
& \Leftrightarrow\left(w_{q}=\sum_{p=1}^{2 R} \Theta_{q, p} v_{p}, \quad q=1, \ldots, 2 R\right) ; \\
\mathbf{A}: H_{1}^{2} \rightarrow H_{2}^{2}, & \mathbf{A}=\mathbf{G}-\mathbf{Z}+\mathbf{K} .
\end{aligned}
$$

With the notation (13)-(16), the system of equations (1)-(3) can be written as

$$
\mathbf{A} \vec{V}=\vec{F}
$$

where $\vec{F}=\left(f_{1}, f_{2}, \ldots, f_{2 R}\right)$.

Lemma 1. The operator $\mathbf{G}: H_{1}^{2} \rightarrow H_{2}^{2}$ is invertible and the operator $\mathbf{G}^{-1}$ : $H_{2}^{2} \rightarrow H_{1}^{2}$ is bounded.

Proof. We introduce the vector-functions:

$$
\begin{gathered}
\vec{X}_{k, q}(\tau)=\left(x_{k, q, 1}(\tau), x_{k, q, 2}(\tau), \ldots, x_{k, q, 2 R}(\tau)\right), \\
x_{k, q, p}=\left\{\begin{array}{ll}
\delta_{q, p} T_{k+1}(\tau), & q=1, \ldots, R \\
\delta_{q, p} T_{k}(\xi), & q=R+1, \ldots, 2 R
\end{array},\right. \\
\vec{Y}_{k, q}(\xi)=\left(y_{k, q, 1}(\xi), y_{k, q, 2}(\xi), \ldots, y_{k, q, 2 R}(\xi)\right), \\
y_{k, q, p}(\xi)= \begin{cases}\delta_{q, p} U_{k}(\xi), & q=1, \ldots, R \\
\delta_{q, p} T_{k}(\xi), & q=R+1, \ldots, 2 R\end{cases}
\end{gathered}
$$

where $\delta_{q, p}$ is a Kronecker delta, and $k=0,1,2, \ldots$

The set of vector functions $\bigcup_{q=0}^{2 R} \bigcup_{k=1}^{\infty} \vec{X}_{k, q}$ is an orthogonal basis in $H_{1}^{2}$ and the set of vector functions $\bigcup_{q=1}^{2 R} \bigcup_{k=0}^{\infty} \vec{Y}_{k, q}$ is an orthogonal basis in $H_{2}^{2}$. Using (9), (13), (18), and (19), we get

$$
\mathbf{G} \vec{X}_{k, q}=\vec{Y}_{k, q}, \quad k=0,1,2, \ldots ; \quad q=1, \ldots, 2 R .
$$

Thus, the operator $\mathbf{G}$ maps the basis of the space $H_{1}^{2}$ onto the basis of the space $H_{2}^{2}$, and $\|\mathbf{G}\|_{H_{1}^{2} \rightarrow H_{2}^{2}}=1$. Therefore, the operator $\mathbf{G}$ is bijective and bounded. So, by the Banach Isomorphism Theorem [16, p. 113] the operator $\mathbf{G}$ has a bounded inverse.

Lemma 2. The operator $\mathbf{A}: H_{\rho,-1}^{2,0} \rightarrow H_{\rho, 1}^{2}$ is invertible and the operator $\mathbf{A}^{-1}$ is bounded. 
Proof. The operator $\mathbf{A}$ is the sum of the invertible operator $\mathbf{G}: H_{1}^{2} \rightarrow H_{2}^{2}$ and the compact operator $-\mathbf{Z}+\mathbf{K}: H_{1}^{2} \rightarrow H_{2}^{2}$. Hence, by virtue of Nikolsky criterion (see [16, p. 150]),

$$
\text { ind }\left(\left.\mathbf{A}\right|_{H_{1}^{2} \rightarrow H_{2}^{2}}\right)=0 \text {. }
$$

From the uniqueness of the problem solutions (1)-(3), it follows that

$$
\operatorname{dim} \operatorname{ker}\left(\left.\mathbf{A}\right|_{H_{1}^{2} \rightarrow H_{2}^{2}}\right)=0 .
$$

Consequently,

$$
\mathbf{A}\left(H_{1}^{2}\right)=H_{2}^{2} \text {. }
$$

Finally, by the Banach Isomorphism Theorem (see [16, p. 113]), the operator A has a bounded inverse.

\section{Approximate Systems of Integral Equations (1)-(3) and Their \\ Properties}

Put

$$
\begin{aligned}
t_{i}^{n} & =\cos \left(\frac{2 i-1}{2 n} \pi\right), & i & =1, \ldots, n ; \\
t_{0, j}^{n} & =\cos \left(\frac{j}{n} \pi\right), & j & =1, \ldots, n-1 .
\end{aligned}
$$

The points $t_{k}^{n}$ are the zeros of Chebychev polynomials of the first kind $\mathrm{T}_{n}(\tau)$ and $t_{0, j}^{n}$ denote the zeros of Chebychev polynomials of the second kind $U_{n-1}(\xi)$.

Define the basis polynomials:

$$
\begin{array}{ll}
l_{1, n-1, i}(\tau)=\frac{1}{n}\left[1+2 \sum_{p=1}^{n-1} \mathrm{~T}_{p}(\tau) \mathrm{T}_{p}\left(t_{i}^{n}\right)\right], & i=1, \ldots, n ; \\
l_{2, n-2, j}(\xi)=\frac{U_{n-1}(\xi)}{U_{n-1}^{\prime}\left(t_{0, j}^{n}\right)\left(\xi-t_{0, j}^{n}\right)}, & j=1, \ldots, n-1 .
\end{array}
$$

They have the properties:

$$
\begin{aligned}
& l_{1, n-1, i}\left(t_{m}^{n}\right)=\delta_{i, m}, \quad i=1, \ldots, n ; \quad m=1, \ldots, n ; \\
& l_{2, n-2, j}\left(t_{0, s}^{n}\right)=\delta_{j, s}, \quad j=1, \ldots, n-1 ; \quad s=1, \ldots, n-1 .
\end{aligned}
$$

We introduce the Lagrange interpolation polynomials:

$$
Q_{q, p, n}(\xi, \tau)=\sum_{j=1}^{n-1} \sum_{i=1}^{n} Q_{q, p}\left(t_{0, j}^{n}, t_{i}^{n}\right) l_{2, n-2, j}(\xi) l_{1, n-1, i}(\tau),
$$




$$
\begin{aligned}
q & =1, \ldots, R ; p=1, \ldots, 2 R ; \\
Q_{q, p, n}(\xi, \tau)=\sum_{j=1}^{n} \sum_{i=1}^{n} Q_{q, p}\left(t_{j}^{n}, t_{i}^{n}\right) l_{1, n-1, j}(\xi) l_{1, n-1, i}(\tau), & \\
q & =R+1, \ldots, 2 R ; p=1, \ldots, 2 R ; \\
f_{q, n}(\xi) & =\sum_{j=1}^{n-1} f_{q}\left(t_{0, j}^{n}\right) l_{2, n-2, j}(\xi), \quad q=1, \ldots, R ; \\
f_{q, n}(\xi) & =\sum_{i=1}^{n} f_{q}\left(t_{i}^{n}\right) l_{1, n-1, i}(\xi), \quad q=R+1, \ldots, 2 R .
\end{aligned}
$$

In order to solve the problem (1)-(3), we consider the following approximate system of integral equations:

$$
\begin{gathered}
\frac{1}{\pi} \int_{-1}^{1} \frac{1}{\tau-\xi} \frac{\nu_{q, n}(\tau) d \tau}{\sqrt{1-\tau^{2}}}+\frac{1}{\pi} \sum_{p=1}^{2 R} \int_{-1}^{1} Q_{q, p, n}(\xi, \tau) \frac{\nu_{p, n}(\tau) d \tau}{\sqrt{1-\tau^{2}}} \\
-\frac{c_{q}}{\pi} \sum_{j=1}^{n-1} \frac{l_{2, n-2, j}(\xi)}{\pi} \int_{-1}^{t_{0, j}^{n}} \frac{\nu_{q, n}(\tau) d \tau}{\sqrt{1-\tau^{2}}}=f_{q, n}(\xi), \\
|\xi|<1, \quad q=1, \ldots, R ; \\
\frac{1}{\pi} \int_{-1}^{1} \frac{\nu_{q, n}(\tau) d \tau}{\sqrt{1-\tau^{2}}}=0, \quad q=1, \ldots, R ; \\
\nu_{q, n}(\xi)-\frac{c_{q}}{\pi} \sum_{i=1}^{n} \sqrt{1-\left(t_{i}^{n}\right)^{2}} l_{1, n-1, i}(\xi) \int_{-1}^{t_{i}^{n}} \frac{\nu_{q-R, n}(\tau) d \tau}{\sqrt{1-\tau^{2}}} \\
+\frac{1}{\pi} \sum_{p=1}^{2 R} \int_{-1}^{1} Q_{q, p, n}(\xi, \tau) \frac{\nu_{p, n}(\tau) d \tau}{\sqrt{1-\tau^{2}}}=f_{q, n}(\xi), \\
|\xi|<1, \quad q=R+1, \ldots, 2 R .
\end{gathered}
$$

The functions $\nu_{q, n}$ are sought in the class of polynomials of degree $n-1$. Subsequently, the reasoning shows the existence of polynomial solutions.

We define the subspaces $L_{\rho, \alpha, n}^{2}$ of the spaces $L_{\rho, \alpha}^{2}$. The elements of these subspaces are polynomials of degree $n$. The subspaces

$$
L_{\rho, \alpha, n}^{2,0}=\left\{u \in L_{\rho, \alpha, n}^{2} \mid(u, 1)_{\alpha}=0\right\}
$$

of the spaces $L_{\rho, \alpha, n}^{2}$ are also taken under consideration. 
Let us define the operators:

$$
\begin{aligned}
& \Theta_{q, p, n}: L_{\rho,-1, n-1}^{2} \rightarrow L_{\rho, 1, n-2}^{2}, \quad\left(\Theta_{q, p, n} u\right)(\xi)=\frac{1}{\pi} \int_{-1}^{1} Q_{q, p, n}(\xi, \tau) \frac{u(\tau) d \tau}{\sqrt{1-\tau^{2}}}, \\
& |\xi|<1 ; \quad i=1, \ldots, R \\
& \Theta_{q, p, n}: L_{\rho,-1, n-1}^{2} \rightarrow L_{\rho,-1, n-1}^{2}, \quad\left(\Theta_{q, p, n} u\right)(\xi)=\frac{1}{\pi} \int_{-1}^{1} Q_{q, p, n}(\xi, \tau) \frac{u(\tau) d \tau}{\sqrt{1-\tau^{2}}} \\
& |\xi|<1 ; \quad i=R+1, \ldots, 2 R ; \\
& \Phi_{n}: L_{\rho,-1, n-1}^{2,0} \rightarrow L_{\rho, 1, n-2}^{2}, \\
& \left(\Phi_{n} u\right)(\xi)=\sum_{j=1}^{n-1} \frac{l_{2, n-2, j}(\xi)}{\pi} \int_{-1}^{t_{0, j}^{n}} \frac{u(\tau) d \tau}{\sqrt{1-\tau^{2}}} \\
& |\xi|<1 \text {; } \\
& \Psi_{n}: L_{\rho,-1, n-1}^{2,0} \rightarrow L_{\rho,-1, n-1}^{2}, \quad\left(\Psi_{n} u\right)(\xi)=\sum_{i=1}^{n} \frac{\sqrt{1-\left(t_{i}^{n}\right)^{2}}}{\pi} l_{1, n-1, j}(\xi) \\
& \times \int_{-1}^{t_{i}^{n}} \frac{u(\tau) d \tau}{\sqrt{1-\tau^{2}}}, \quad|\xi|<1
\end{aligned}
$$

We introduce the subspaces $H_{1, n}^{2}$ of $H_{1}^{2}$ and the subspaces $H_{2, n}^{2}$ of $H_{2}^{2}$ :

$$
\begin{aligned}
\vec{V}_{n} \in H_{1, n}^{2} \Leftrightarrow\left(v_{q, n} \in L_{\rho,-1, n-1}^{2,0},\right. & q=1, \ldots, R ; \\
v_{q, n} \in L_{\rho,-1, n-1}^{2}, & q=R+1, \ldots, 2 R) ; \\
\vec{W}_{n} \in H_{2, n}^{2} \Leftrightarrow\left(w_{q, n} \in L_{\rho, 1, n-2}^{2},\right. & q=1, \ldots, R ; \\
w_{q, n} \in L_{\rho,-1, n-1}^{2}, & q=R+1, \ldots, 2 R) .
\end{aligned}
$$

Consider the operators:

$$
\begin{aligned}
& \mathbf{Z}_{n}: H_{1, n}^{2} \rightarrow H_{2, n}^{2}, \quad\left(\vec{W}_{n}=\mathbf{Z}_{n} \vec{V}_{n}\right) \\
& \Leftrightarrow\left\{\begin{array}{ll}
w_{q, n}=c_{q} \Phi_{n} v_{q, n}, & q=1, \ldots, R ; \\
w_{q, n}=c_{q} \Psi_{n} v_{q-R, n}, & q=R+1, \ldots, 2 R
\end{array}\right\} ; \\
& \mathbf{K}_{n}: H_{1, n}^{2} \rightarrow H_{2, n}^{2}, \quad\left(\vec{W}_{n}=\mathbf{K}_{n} \vec{V}_{n}\right) \\
& \Leftrightarrow\left(w_{q, n}=\sum_{p=1}^{2 R} \Theta_{q, p, n} t v_{p, n}, \quad q=1, \ldots, 2 R\right) ;
\end{aligned}
$$




$$
\mathbf{A}_{n}: H_{1, n}^{2} \rightarrow H_{2, n}^{2}, \quad \mathbf{A}_{n}=\mathbf{G}-\mathbf{Z}_{n}+\mathbf{K}_{n} .
$$

With the notation (28)-(30), the system of equations (21)-(23) can be written as

$$
\mathbf{A}_{n} \vec{V}_{n}=\vec{F}_{n}
$$

where $\vec{F}_{n}=\left(f_{1, n}, f_{2, n}, \ldots, f_{2 R, n}\right)$.

For all $n \geq 4$, the following estimates hold true [15]:

$$
\begin{aligned}
\left\|f_{q, n}-f_{q}\right\|_{L_{\rho, 1}^{2}} & \leq \frac{M_{1}}{\sqrt{n}}, \quad q=1, \ldots, R ; \\
\left\|f_{q, n}-f_{q}\right\|_{L_{\rho,-1}^{2}} & \leq \frac{M_{1}}{\sqrt{n}}, \quad q=R+1, \ldots, 2 R ; \\
\left\|\Theta_{q, p, n}-\Theta_{q, p}\right\|_{L_{\rho,-1}^{2} \rightarrow L_{\rho, 1}^{2}} & \leq \frac{M_{2}}{\sqrt{n}}, \quad q=1, \ldots, R ; \quad p=1, \ldots, 2 R ; \\
\left\|\Theta_{q, p, n}-\Theta_{q, p}\right\|_{L_{\rho,-1}^{2} \rightarrow L_{\rho,-1}^{2}} & \leq \frac{M_{2}}{\sqrt{n}}, \quad q=R+1, \ldots, 2 R ; \quad p=1, \ldots, 2 R,
\end{aligned}
$$

where

$$
\begin{aligned}
& M_{1}=24 \sqrt{2 \pi} \max _{q=1, \ldots, 2 R}\left\|f_{q}\right\|_{C[-1,1]}, \\
& M_{2}=48 \sqrt{2} \pi \max _{q=1, \ldots, 2 R}\left\|Q_{q, p}\right\|_{C([-1,1] \times C[-1,1])} .
\end{aligned}
$$

These estimates are the consequences of Jackson's Theorems (see Corollary 1 of Theorem 2 in [17, p. 128]).

Lemma 3. For all natural $n$, the inequality

$$
\left\|\mathbf{A}-\mathbf{A}_{n}\right\|_{H_{1, n}^{2} \rightarrow H_{2}^{2}} \leq \frac{M^{*}}{\sqrt{n}}
$$

holds true. Besides,

$$
\left\|\mathbf{A}-\mathbf{A}_{n}\right\|_{H_{1, n}^{2} \rightarrow H_{2}^{2}} \rightarrow 0, \quad n \rightarrow \infty .
$$

Proof. The following inequality clearly holds:

$$
\left\|\mathbf{A}-\mathbf{A}_{n}\right\|_{H_{1, n}^{2} \rightarrow H_{2}^{2}} \leq\left\|\mathbf{Z}_{n}-\mathbf{Z}\right\|_{H_{1, n}^{2} \rightarrow H_{2}^{2}}+\left\|\mathbf{K}_{n}-\mathbf{K}\right\|_{H_{1, n}^{2} \rightarrow H_{2}^{2}} .
$$

The set of vector functions

$$
E_{1, n}=\left(\bigcup_{q=1}^{R} \bigcup_{k=0}^{n-2} \vec{X}_{k, q}\right) \bigcup\left(\bigcup_{q=R+1}^{2 R} \bigcup_{k=0}^{n-1} \vec{X}_{k, q}\right)
$$


is an orthogonal basis in $H_{1, n}^{2}$ and the set of vector functions

$$
E_{2, n}=\left(\bigcup_{q=1}^{R} \bigcup_{k=0}^{n-2} \vec{Y}_{k, q}\right) \bigcup\left(\bigcup_{q=R+1}^{2 R} \bigcup_{k=0}^{n-1} \vec{Y}_{k, q}\right)
$$

is an orthogonal basis in $H_{2, n}^{2}$. Let us note that $\operatorname{dim}\left(H_{1, n}^{2}\right)=\operatorname{dim}\left(H_{2, n}^{2}\right)=$ $R(2 n-3)$.

The estimations

$$
\frac{\left\|\left(\mathbf{K}-\mathbf{K}_{n}\right) \vec{X}_{k, q}\right\|_{H_{2}^{2}}}{\left\|\vec{X}_{k, q}\right\|_{H_{1}^{2}}} \leq \frac{2 M_{2} R}{\sqrt{n}}
$$

hold true for all $\vec{X}_{k, q} \in E_{1, n}$. Inequality (39) is the consequence of estimates (34) and (35). Furthermore,

$$
\left\|\left(\mathbf{K}-\mathbf{K}_{n}\right)\right\|_{H_{1, n}^{2} \rightarrow H_{2}^{2}} \leq \frac{2 M_{2} R}{\sqrt{n}} .
$$

Let $u_{n}(\tau)$ be a polynomial of degree $(n-1)$ with the property $\left(u_{n}, 1\right)_{-1}=0$. Properties (10), (11) of the operators $\Phi$ and $\Psi$ imply that the function $\Phi u_{n} \in$ $C^{0, \frac{1}{2}}$ and $\Psi u_{n} \in L_{\rho, 1, n}^{2}$.

Reasoning as in [15, p. 60], we get

$$
\begin{aligned}
\left\|\Phi-\Phi_{n}\right\|_{L_{\rho,-1, n-1}^{2} \rightarrow L_{\rho, 1}^{2}} & \leq \frac{24}{\sqrt{n}} \\
\left\|\Psi-\Psi_{n}\right\|_{L_{\rho,-1, n-1}^{2} \rightarrow L_{\rho,-1}^{2}} & \leq \frac{24}{\sqrt{n}}, \quad \forall u_{n} \in L_{\rho,-1, n-1}^{2,0} .
\end{aligned}
$$

Hence, estimations (41) show that the operators

$$
\Phi_{n}: L_{\rho,-1, n-1}^{2,0} \rightarrow L_{\rho, 1, n-2}^{2}, \quad \Psi_{n}: L_{\rho,-1, n-1}^{2,0} \rightarrow L_{\rho,-1, n-1}^{2}
$$

are bounded.

Taking into account (14) and (28), we obtain

$$
\left\|\left(\mathbf{Z}-\mathbf{Z}_{n}\right) \vec{X}_{k, q}\right\|_{H_{1}^{2}}=0, \quad q=R+1, \ldots, 2 R,
$$

and

$$
\left\|\left(\mathbf{Z}-\mathbf{Z}_{n}\right) \vec{X}_{k, q}\right\|_{H_{1}^{2}} \leq\left|c_{q}\right|\left\|\left(\Phi-\Phi_{n}\right) T_{k}\right\|_{L_{\rho,-1}^{2} \rightarrow L_{\rho, 1}^{2}}
$$




$$
+\left|c_{q+R}\right|\left\|\left(\Psi-\Psi_{n}\right) T_{k}\right\|_{L_{\rho,-1}^{2} \rightarrow L_{\rho,-1}^{2}}, \quad q=1, \ldots, R
$$

Moreover,

$$
\left\|\mathbf{Z}-\mathbf{Z}_{n}\right\|_{H_{1, n}^{2} \rightarrow H_{2}^{2}}=\max _{\vec{X}_{k, q} \in E_{1, n}} \frac{\left\|\left(\mathbf{Z}-\mathbf{Z}_{n}\right) \vec{X}_{k, q}\right\|_{H_{2}^{2}}}{\left\|\vec{X}_{k, q}\right\|_{H_{1}^{2}}} \leq \frac{48 C}{\sqrt{n}},
$$

where $C=\max _{q=1, \ldots, 2 R}\left|c_{q}\right|$. Finally, the validity of Lemma 3 follows from (38), (40), and (43), where $M^{*}=2 R M_{2}+48 C$.

In [18], the following theorem can be found.

Theorem 1. Let $X$ and $Y$ be normed linear spaces and let $\widetilde{X} \subset X$ and $\tilde{Y} \subset Y$ be finite-dimensional subspaces of the same dimension. We consider two equations.

The equation for the exact solution of the problem

$$
A u=f, \quad u \in X, \quad f \in Y,
$$

and the equation for the approximate solution of the problem

$$
\widetilde{A} \widetilde{u}=\widetilde{f}, \quad \widetilde{u} \in \widetilde{X}, \quad \widetilde{f} \in \widetilde{Y},
$$

where $A$ and $\widetilde{A}$ are the linear operators

Assume that

1) the operator $A$ is invertible and the operator $A^{-1}: Y \rightarrow X$ is bounded,

2) the inequality

$$
p=\left\|A^{-1}\right\|_{Y \rightarrow X}\|A-\widetilde{A}\|_{\widetilde{X} \rightarrow Y}<1
$$

holds.

Then

1) for any function $\tilde{f} \in \widetilde{Y}$, the equation (45) has the unique solution $\widetilde{u}^{*} \in \widetilde{X}$;

2) let $u^{*} \in X$ be the solution of equation (44) and let $\delta=\|f-\widetilde{f}\|_{Y}$, then

$$
\left\|u^{*}-\widetilde{u}^{*}\right\| \leq\left\|A^{-1}\right\|_{Y \rightarrow X}(1-p)^{-1}\left(\delta+p\|f\|_{Y}\right) .
$$

Lemmas 1-3 and Theorem 1 lead us to the following result. 
Theorem 2. Let us denote

$$
M=\left(2 M^{*}\left\|\mathbf{A}^{-1}\right\|_{H_{2}^{2} \rightarrow H_{1}^{2}}\right)^{2} .
$$

For all natural $n>M$, the following statements hold true.

1. Problems (31) have unique solutions.

2. The vector-functions $\vec{V}_{n} \in H_{1, n}^{2}$.

3. The sequence $\left\{\vec{V}_{n}\right\}_{n=[M]+1}^{\infty}$ of the approximate solutions of problems (31) converges to the exact solution of problem (17) in the norm of the space $H_{1}^{2}$. Moreover,

$$
\left\|\vec{V}-\vec{V}_{n}\right\|_{H_{1}^{2}} \leq \frac{M^{* *}}{\sqrt{n}}
$$

Proof. Let us define the numbers

$$
p_{n}=\left\|\mathbf{A}-\mathbf{A}_{n}\right\|_{H_{1, n}^{2} \rightarrow H_{2}^{2}}\left\|\mathbf{A}^{-1}\right\|_{H_{2}^{2} \rightarrow H_{1}^{2}} .
$$

It follows from estimation (36) and (46) that $p_{n} \leq \frac{1}{2}$ for $n>M$.

Appealing to Theorem 1 and the previous estimation concludes the uniqueness and existence of solutions of problems (31), where $\vec{V}_{n} \in H_{1, n}^{2}$. Also, the estimations

$$
\begin{aligned}
\left\|\vec{V}-\vec{V}_{n}\right\|_{H_{1}^{2}} \leq\left\|\mathbf{A}^{-1}\right\|_{H_{2}^{2} \rightarrow H_{1}^{2}}\left(1-p_{n}\right)^{-1} & \\
& \times\left(\left\|\vec{F}_{n}-\vec{F}\right\|_{H_{2}^{2}}+p_{n}\|\vec{F}\|_{H_{1}^{2}}\right), \quad n>M,
\end{aligned}
$$

follow from the statements of Theorem 1. Thus, inequality (47) holds true for

$$
M^{* *}=2\left\|\mathbf{A}^{-1}\right\|_{H_{2}^{2} \rightarrow H_{1}^{2}}\left(2 R M_{1}+M^{*}\left\|\mathbf{A}^{-1}\right\|_{H_{2}^{2} \rightarrow H_{1}^{2}}\|\vec{F}\|_{H_{1}^{2}}\right) .
$$

We obtain the value of $M^{* *}$ as a direct consequence of estimations (32), (33), (36), and (49) . This completes the proof of Theorem 2.

Corollary 1. For all natural $n>M$, the following statements hold true.

1. The systems of integral equations (21)-(23) have a unique solution, where the functions $v_{q, n}$ are the polynomials of degree $(n-1)$.

2. For all $q=1, \ldots, M$, the sequences $\left\{v_{q, n}\right\}_{n=[M]+1}^{\infty}$ converge to the functions $v_{q}$, which are the exact solutions of problem (1)-(3) in the norm of the space $L_{\rho,-1}^{2}$. Moreover,

$$
\left\|v_{q, n}-v_{q}\right\|_{L_{\rho,-1}^{2}} \leq \frac{M^{* *}}{\sqrt{n}}, \quad \forall n \in N, \quad n>M
$$

Journal of Mathematical Physics, Analysis, Geometry, 2017, Vol. 13, No. 3 


\section{Conclusions}

The justification of the method of approximate solution of the boundary integral equations considered in [19] was given. The results obtained in the paper can be used as basic solutions of other problems considered in [20-22].

\section{Acknowledgements}

The author is grateful to Professor Yu.V. Gandel for constant attention to this work and for useful discussions.

\section{References}

[1] A.S. Il'insky, A.Ja. Slepjan and G.Ja. Slepjan, Propagation, Diffraction and Dissipation of Electromagnetic Waves, The IEE and Peter Peregrinous Ltd., Electromagnetic Waves (Series 36), London, UK, 1993.

[2] N.I. Akhiezer, Lectures on Integral Transforms. Translations of Mathematical Monographs, 70, Amer. Math. Soc., Providence, RI, 1988.

[3] Yu.V. Gandel, Parametric Representations of Integral and Psevdodifferential Operators in Diffraction Problems, Proc. 10th Int. Conf. on Math. Methods in Electromagnetic Theory, Dnepropetrovsk, Ukraine, Sept. 14-17, 2004, 57-62.

[4] Yu.V. Gandel', Boundary-Value Problems for the Helmholtz Equation and their Discrete Mathematical Models, J. Math. Sci. 171 (1990), No. 1, 74-88.

[5] Yu.V. Gandel and V.D. Dushkin, The Method of Parametric Representations of Integral and Pseudo-differential Operators in Diffraction Problems on Electrodynamic Structures, Proceedings of the International Conference Days on Diffraction DD 2012, 28 May-1 June, 2012, 76-81.

[6] Yu.V. Gandel and V.D. Dushkin, Mathematical Models of Two-Dimensional Diffraction Problems: Singular Integral Equations and Numerical Methods of Discrete Singularities Method, Academy of IT of the MIA of Ukraine, Kharkiv, 2012 (Russian).

[7] S.M. Belotserkovsky and I.K. Lifanov, Method of Discrete Vortices, CRC Press, New York, 1993.

[8] I.K. Lifanov, Singular Integral Equations and Discrete Vortices, VSP, Utrecht, Netherlands, Tokyo, Japan, 1996.

[9] Yu.V. Gandel' and T.S. Polyanskaya, Justification of a Numerical Method for Solving Systems of Singular Integral Equations in Diffraction Grating Problems, Differ. Equ. 39 (2003), I. 9, 1295-1307.

[10] Yu.V. Gandel', S.V. Eremenko, and T.S. Polyanskaya, Mathematical Problems in the Method of Discrete Currents. Justification of the Numerical Method of Discrete Singularities of Solutions of Two-Dimensional Problems of Diffraction of Electromagnetic Waves, Educational aid. Part II, Kharkov State University, Kharkov, 1992 (Russian). 
[11] V.D. Dushkin, The Justification of Numerical Solution of Boundary Integral Equations of Wave Scattering Problems on Impedance Lattice, Visn. Kharkiv. Nats. Univ. No. 1120, Mat. Prikl. Mat. Mekh. (2014), Issue 69, 20-28.

[12] Yu.V. Gandel' and V.D. Dushkin, The Approximate Method for Solving the Boundary Integral Equations of the Problem of Wave Scattering by Superconducting Lattice, Am. J. App. Mathematics and Statistics 2 (2014), No. 6, 369-375.

[13] Yu.V. Gandel' and V.D. Dushkin, The Boundary Integral Equations of the Third Boundary-Value Problem for the Helmholtz Equation in the $R_{+}^{2}$ with Plane-Parallel Slits, Dopov. Nats. Akad. Nauk Ukr. 8 (2014), 14-19 (Russian).

[14] Yu.V. Gandel', V.F. Kravchenko, and V.I. Pustovoit, Scattering of Electromagnetic Waves by a Thin Superconducting Band, Dokl. Math. 54 (1996), No. 3, 959-961.

[15] Yu.V. Gandel', Introduction to Methods of Evaluation of Singular and Hypersingular Integrals, Izd. Kharkov. Nats. Univ., Kharkov, 2002 (Russian).

[16] S.S. Kutateladze, Fundamentals of Functional Analysis, Kluwer Academic Publishers Group, Dordrecht, Netherlands, 1996.

[17] I.P. Natanson, Constructive Function Theory, 1, Frederic Ungar Puplishing Co., New York, 1964.

[18] B.G. Gabdulkhaev, The Optimal Approximation of Solutions of Linear Problems, Kazan. Univ. Publishing, Kazan, 1980 (Russian).

[19] Yu.V. Gandel' and G.L. Sidel'nikov, The Method of Integral Equations in the Third Boundary-Value Problem of Diffraction on a Bounded Grating Over a Flat Screen, Differ. Equ. 35 (1999), No. 9, 1169-1175.

[20] Yu.V. Gandel' and V.D. Dushkin, Mathematical Model of Polarized Wave Scattering on Impedance Strips located on Screened Dielectric Layer, Mat. Metodi Fiz.-Mekh. Polya 57 (2014), No. 1, 125-132.

[21] Yu.V. Gandel' and V.D. Dushkin, Mathematical Model of Polarized Wave Scattering on Impedance Strips located on Screened Dielectric Layer, J. Math. Sci. 212 (2016), No. 2, 156-166.

[22] V.D. Dushkin, Mathematical Models of Plane Wave Scattering on Multilayer Impedance Structures, Visn. Lviv. Univ. Prikl. Mat. Inform. No. 20 (2013), 6976. 